\title{
Are low-carbohydrate or low-fat diets effective in reducing adult acne?
}

\author{
Dams, Travis MD; Norkus, Bryan MD; Williams, Ashley MD; Williams, Jeff DO
}

\section{EVIDENCE-BASED ANSWER}

A low glycemic load diet compared with a high glycemic load diet reduces inflammatory acne lesion counts, but not noninflammatory lesion counts (SOR: B, meta-analysis of small randomized controlled trials). Patients with moderate to severe acne report consuming foods with a higher average glycemic index, as well as higher sugar, fat, and milk intake, but lower fish intake than patients with no acne (SOR: B, cross-sectional study and case-control study).

A 2015 meta-analysis of 35 randomized controlled trials (RCTs) $(\mathrm{N}=3,227)$ examined the use of any kind of complementary and alternative medicine, including diet, in the treatment of acne. ${ }^{1}$ Two RCTs $(\mathrm{N}=86)$ examined the effect of low glycemic load diets $(25 \%$ of energy from protein, $45 \%$ from low glycemic index carbohydrates, and 30\% from fat) compared with high glycemic load diets on acne lesion counts, but the definitions of low versus high glycemic load were not reported. Patients were all 20 to 30 years old and had had acne for three months to seven years. At 31 to 90 days, a low glycemic load diet compared with a high glycemic load diet showed reduced inflammatory lesion counts (one trial; $\mathrm{N}=53$; mean difference $[\mathrm{MD}]-7.6$ lesions; $95 \% \mathrm{CI},-13.5$ to -1.7 ) and total lesion count (one trial; $\mathrm{N}=53$; $\mathrm{MD}$ 8.0 lesions; $95 \% \mathrm{CI},-14.9$ to -1.3 ). No reduction was observed in noninflammatory lesions (two trials; $\mathrm{N}=75 ; \mathrm{MD}-3.9 ; 95 \% \mathrm{CI},-10.1$ to 2.3$)$.

This is the authors' manuscript of the work published in final form as:

Dams, T., Norkus, B., Williams, A., \& Williams, J. (2020). Are low-carbohydrate or low-fat diets effective in reducing adult acne? Evidence-Based Practice, 23(1), 31. https://doi.org/10.1097/EBP.0000000000000433 
A 2014 cross-sectional study of 248 young adults 18 to 25 years old from New York City examined the relationship between self-reported dietary factors and perceived acne. ${ }^{2}$ Participants completed a dietary questionnaire to estimate the glycemic index of food they consumed and the amount of milk, fish, sugars, and fats consumed. They also completed a self-reported acne severity questionnaire based on dermatologist-approved acne categories (no acne, mild acne, or moderate to severe acne). Participants with moderate to severe acne reported a higher average glycemic index of the foods they consumed (52 vs 49; $P<.001$ ), a higher daily sugar intake (199 vs $56 \mathrm{~g} ; P<.001$ ), a higher daily saturated fat intake (31 vs $16 \mathrm{~g} ; P<.001)$, a higher daily trans-fat intake (9.8 vs $2.4 \mathrm{~g} ; P<.001)$, more daily 8 oz milk servings $(0.7$ vs $0.3 ; P<.001)$, and less daily $3 \mathrm{oz}$ fish servings $(0.2 \mathrm{vs} 0.7 ; P=.0002)$, all compared with participants without acne.

A 2012 case-control study evaluated the glycemic load and consumption of milk and ice cream in 44 Malaysian patients 18 to 30 years old with acne compared with 44 matched patients without acne. ${ }^{3}$ All participants completed a three-day food diary, and the glycemic load was calculated for each participant by multiplying the glycemic index of each food item consumed by its carbohydrate content in grams and dividing by 100 . Patients with acne had a significantly higher dietary glycemic load compared with matched individuals without acne (175 vs $122 ; P<.001)$. Consumption of milk at least once per week increased the risk of having acne compared with those who do not (odds ratio 4.0, 95\% CI, 1.4-11). Limitations of this study included limited dietary data from participants, small study size, and unknown glycemic indices of some Malaysian foods. 


\section{References}

1. Cao H, Yang G, Wang Y, et al. Complementary therapies for acne vulgaris (review). Cochrane Database Syst Rev. 2015; (1):CD009436. [STEP 1]

2. Burris J, Rietkerk W, Woolf K. Relationships of self-reported dietary factors and perceived acne severity in a cohort of New York young adults. J Acad Nutr Diet. 2014; 114:384-392. [STEP 4]

3. Ismail N, Manaf Z, Azizan N. High glycemic load diet, milk and ice cream consumption are related to acne vulgaris in Malaysian young adults: a case control study. BMC Dermatol. 2012; 12:13. [STEP 4] 